\title{
Rapport de gestion électronique 2015: une démarche responsable
}

\section{Anne-Geneviève Bütikofer}

lic. iur., secrétaire générale de la FMH

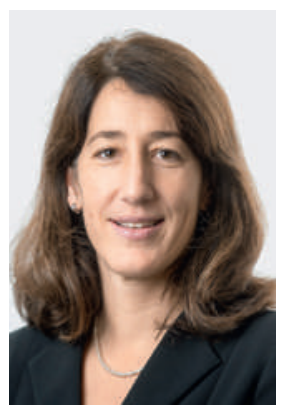

Selon le même calendrier, conformément aux années précédentes, j'ai le plaisir de vous annoncer la publication, respectivement la mise à disposition du rapport de gestion 2015 de la FMH. Cependant, contrairement aux années précédentes, plus aucun exemplaire n'a été imprimé sur papier. L'exemplaire auquel non seulement tous les membres de la FMH, mais également tous les partenaires ont dorénavant accès est une version exclusivement électronique.

Ce rapport de gestion, sous forme de ePaper, n'est pourtant qu'une version transitoire, avant que vous n'ayez accès, au printemps 2017, à notre rapport de gestion électronique en ligne et dans sa version définitive et interactive sur le portail de la FMH. Dans la version ePaper 2015, publiée ce jour sous le titre «Les soins de santé en Suisse - aujourd'hui et demain", vous retrouverez les rubriques qui vous sont désormais familières, respectivement la rétrospective de l'activité de la FMH, du Comité central, de son Secrétariat général et de ses divisions, les rapports des mandats internationaux, un aperçu de la statistique médicale, mais également les comptes annuels intégrant nouvellement un rapport de situation selon les exigences légales en matière de présentation des comptes. Enfin, le débat public, dont nous vous donnons un aperçu dans ce même numéro du BMS (p. 494), réunit la conseillère nationale Ruth Humbel Näf, le CEO de Medbase, Marcel Napierala, et le président de la FMH, Jürg Schlup, pour un échange passionnant placé sous l'intitulé du rapport de gestion.

\section{Le débat du rapport de gestion est un échange} passionnant sous l'intitulé «Les soins de santé en Suisse - aujourd'hui et demain».

Si la logique de la suppression du support papier a été en premier lieu dictée par un motif économique, au-delà de la pure logique d'épargne elle s'inscrit également dans une volonté de responsabilité sociale et de développement durable. En effet, la FMH est une association responsable et son secrétariat général entend appliquer les principes de Responsabilité Sociale d'Entre- prise (RSE), qui invitent à mieux prendre en compte les impacts sociaux, environnementaux et économiques dans le cadre de notre activité. Aussi, considérer les étapes de restructuration financière, que vient de franchir la FMH, comme des pures mesures de stabilisation budgétaire reviendrait à émettre une vision réductrice de la gestion du changement et du développement que poursuit la FMH. En effet, notre démarche vise une action durable, efficace et en conformité avec les valeurs qui sont les nôtres.

Naturellement, la suppression du rapport de gestion sous sa forme papier n'est pas la seule mesure conforme à une RSE qui a été mise en place dans le cadre de la stabilisation budgétaire, ou au cours de ces

\section{La suppression du support papier s'inscrit} également dans une volonté de responsabilité sociale et de développement durable.

dernières années. De manière générale, la RSE s'étend à de nombreux thèmes dont nous tenons compte dans le cadre de notre activité et dans nos interactions avec nos collaborateurs, nos membres et nos partenaires. On peut mentionner par exemple, entre autres, le renforcement des mesures de gestion, la formalisation des conditions de travail ainsi que la gestion de la santé en entreprise (GSE) pour le Secrétariat général, l'introduction de la gestion du risque et de la protection des données, la défense des intérêts de nos membres au travers du recentrage de nos prestations, la révision de nos principes de gestion financière et la transparence.

De nombreux efforts peuvent être et seront encore menés pour améliorer nos changements de pratique et nous inscrire définitivement dans un effort de participation au développement durable. Ainsi, nous pourrons continuer d'apporter une contribution permettant de répondre aux défis auxquels fait face notre société. Aussi en lisant notre rapport de gestion électronique et en prenant électroniquement connaissance des événements clés qui ont jalonné l'année 2015, vous participez à cet effort. Je vous souhaite ainsi une lecture passionnante et responsable. 